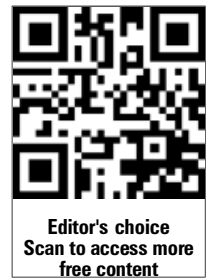

\begin{abstract}
${ }^{1}$ National Health and Medical Research Council of Australia Centre of Research Excellence to Reduce Inequality in Heart Disease/Preventative Health, Baker IDI Heart and Diabetes Institute, Melbourne, Victoria, Australia

${ }^{2}$ Department of Epidemiology and Preventative Medicine

Monash University, Melbourne, Victoria, Australia
\end{abstract}

\section{Correspondence to} Professor Simon Stewart, National Health and Medical Research Council of Australia Centre of Research Excellence to Reduce Inequality in Heart Disease/Preventative Health, Baker IDI Heart and Diabetes Institute, PO Box 6492, St Kilda Road Central, Melbourne, VIC 8008 Australia;

Simon.Stewart@bakeridi. edu.au

Received 9 October 2012 Revised 3 December 2012 Accepted 10 December 2012 Published Online First 12 January 2013

\footnotetext{
To cite: Ball J,

Carrington MJ, Stewart $\mathrm{S}$

et al. Heart

2013;99:542-547.
}

\title{
Mild cognitive impairment in high-risk patients with chronic atrial fibrillation: a forgotten component of clinical management?
}

\author{
Jocasta Ball, ${ }^{1,2}$ Melinda Jane Carrington, ${ }^{1,2}$ Simon Stewart, ${ }^{1,2}$ \\ on behalf of the SAFETY investigators
}

\section{ABSTRACT \\ Objective We examined cognitive function in older hospitalised patients with chronic atrial fibrillation (AF). \\ Design A prospective substudy of a multicentre randomised trial of an AF-specific disease management intervention (the Standard versus Atrial Fibrillation spEcific managmenT studY; SAFETY).}

Setting Three tertiary referral hospitals within Australia. Patients A total of 260 patients with chronic AF: mean age $72 \pm 11$ years, $53 \%$ men, mean $\mathrm{CHA}_{2} \mathrm{DS}_{2}$ VASc score $4 \pm 2$.

Interventions Cognitive function was assessed at baseline (during inpatient stay) using the Montreal Cognitive Assessment (MoCA).

Main Outcome Measures The extent of mild cognitive impairment ( $\mathrm{MCl}$-defined as a MoCA score $<26)$ in AF patients and identification of independent predictors of $\mathrm{MCl}$.

Results Overall, 169 patients (65\%, 95\% Cl 59\% to $71 \%$ ) were found to have $\mathrm{MCl}$ at baseline (mean MoCA score $21 \pm 3$ ). Multiple deficits in cognitive domains were identified, most notably in executive functioning, visuospatial abilities and short-term memory. Predictors of $\mathrm{MCl}$ (age and sex-adjusted) were lower education level (technical/trade school level OR 6.00,95\% Cl 2.07 to $17.42 ;<8$ years school education OR $5.29,95 \% \mathrm{Cl}$ 1.95 to 14.36 vs $8-13$ years), higher $\mathrm{CHA}_{2} \mathrm{DS}_{2}$-VASC score (OR 1.46, 95\% Cl 1.23 to 1.74) and prescribed digoxin (OR 2.19, 95\% Cl 1.17 to 4.10 ).

Conclusions $\mathrm{MCl}$ is highly prevalent amongst typically older high-risk patients hospitalised with AF. Routine assessment of cognitive function with adjustment of clinical management is indicated for this patient group.

\section{BACKGROUND}

With a reported population prevalence of $1-2 \%$, atrial fibrillation (AF) is the most common sustained cardiac arrhythmia seen in clinical practice. ${ }^{1}$ The numbers of cases are predicted at least to double by $2050^{1}$ as the key pathways to AF (including advancing age, chronic forms of cardiovascular disease, hypertension and obesity/metabolic disorders) reach historically high levels. From an individual perspective, AF (in all its forms) confers an independent 1.52 -fold probability of death in the longer term ${ }^{2}$ with close links to ischaemic stroke (3-5-fold risk ${ }^{3}$ ), heart failure (up to $50 \%$ develop $\mathrm{AF})^{4}$ and acute coronary syndromes (AF (usually paroxysmal) is documented in almost $25 \%$ of patients who present to hospital during ischaemic/postischaemic episodes). ${ }^{5}$ As such, there is a challenge to improve clinical management and subsequent health outcomes.

Despite a growing body of literature, an often forgotten component of the burden of AF, with important clinical implications, is cognitive impairment and vascular dementia. In fact, the prevalence and influence of cognitive impairment is considerable across a wide spectrum of cardiac diseases, with deficits most notably identified in executive functioning. ${ }^{6}$ A number of studies (predominantly using the mini-mental state examination; MMSE) have identified $\mathrm{AF}$ as an independent predictor of cognitive impairment. ${ }^{7-9}$ Moreover, the prevalence of cognitive impairment in both community dwelling and hospitalised individuals with $\mathrm{AF}$ is reported to be approximately 26-51\%. ${ }^{8}$ Although the pathways to cognitive impairment in AF are not fully understood, in those individuals with AF suffering from concurrent stroke or transient ischaemic attack (TIA), $15-26 \%$ have signs of one or more silent cerebral infarcts on computed tomography. ${ }^{10}{ }^{11}$ Furthermore, AF is regarded as a common cause of white matter low attenuation, most likely due to cerebral hypoperfusion. ${ }^{12}$ In comparison, population-based studies of 'healthy' individuals aged 60-64 years suggest the underlying prevalence of cognitive impairment is a far lower figure of approximately $4 \% .^{13}$ In healthy individuals older than 65 years of age, equivalent prevalence estimates rise to $10-20 \%$. $^{14}$

Due to the clinical heterogeneity and treatment complexities of AF, careful and balanced management is required to prevent known adverse effects. Individuals with $\mathrm{AF}$ and even forms of mild cognitive impairment (MCI) should be considered at higher risk of poorer health outcomes due to the potentially complex requirements of self-managing therapies with narrow therapeutic margins. As such, strict and effective adherence to clinical routines and required treatments is influenced by MCI, potentially impairing a patient's capacity to plan, sequence and carry out tasks associated with $\mathrm{AF}$ management/self-care, diminishing a patient's ability to participate in decisions surrounding their medical care and/or determine if they are at high risk of a serious clinical event (eg, a haemorrhagic stroke).

\section{STUDY OBJECTIVE}

We sought to determine the extent of MCI in a cohort of typically older and non-demented 
patients hospitalised with a diagnosis of chronic AF. We hypothesised that the prevalence of MCI would be high in this cohort.

\section{METHODS}

\section{Study setting}

As part of a systematic approach to risk delineation and optimising the management of hospitalised patients with chronic forms of AF, we are undertaking the Standard versus Atrial Fibrillation spEcific managemenT studY (SAFETY). ${ }^{15}$ The overall purpose and design of SAFETY has been described in greater detail previously. ${ }^{15}$ In brief, 335 non-demented patients with chronic forms of AF (see table 1) were randomly assigned into this study and all were subject to comprehensive baseline profiling. Patients were randomly assigned to either usual post-discharge

Table 1 Summary of cognitive function in SAFETY participants at baseline (in hospital)

\begin{tabular}{|c|c|c|c|c|}
\hline & $\begin{array}{l}\text { Total } \\
(n=260)\end{array}$ & $\begin{array}{l}\mathrm{MCl} \\
(n=169)\end{array}$ & $\begin{array}{l}\text { No MCl } \\
(n=91)\end{array}$ & $\begin{array}{l}p \\
\text { Value }\end{array}$ \\
\hline Mean age (years) & $72 \pm 11$ & $74 \pm 10$ & $68 \pm 12$ & $<0.001$ \\
\hline Aged <65 years (\%) & $68(26 \%)$ & $34(20 \%)$ & $34(37 \%)$ & $<0.01$ \\
\hline Aged $65-74$ years (\%) & $79(30 \%)$ & $50(30 \%)$ & $29(32 \%)$ & 0.70 \\
\hline Aged $\geq 75$ years $(\%)$ & $113(44 \%)$ & $85(50 \%)$ & $28(31 \%)$ & $<0.01$ \\
\hline Male sex (\%) & 137 (53\%) & $89(53 \%)$ & $48(53 \%)$ & 0.99 \\
\hline $\begin{array}{l}\text { Mean } \mathrm{CHA}_{2} \mathrm{DS}_{2} \text {-VASC } \\
\text { score }( \pm \mathrm{SD})\end{array}$ & $4 \pm 2$ & $4 \pm 2$ & $3 \pm 2$ & $<0.001$ \\
\hline $\begin{array}{l}\text { Education < secondary } \\
\text { school* }^{*}\end{array}$ & $80(31 \%)$ & $65(39 \%)$ & $15(17 \%)$ & $<0.001$ \\
\hline $\begin{array}{l}\text { Mean MoCA score } \\
( \pm S D)\end{array}$ & $23 \pm 4$ & $21 \pm 3$ & $27 \pm 1$ & $<0.001$ \\
\hline \multicolumn{5}{|l|}{ AF subtype } \\
\hline Paroxysmal (\%) & $10(4 \%)$ & $6(4 \%)$ & $4(4 \%)$ & 0.74 \\
\hline Persistent (\%) & $224(86 \%)$ & $147(87 \%)$ & 77 (85\%) & 0.60 \\
\hline Permanent (\%) & $26(10 \%)$ & $16(10 \%)$ & $10(11 \%)$ & 0.70 \\
\hline \multicolumn{5}{|l|}{ Comorbidities } \\
\hline Hypertension (\%) & $188(72 \%)$ & $128(76 \%)$ & $60(66 \%)$ & 0.09 \\
\hline CAD (\%) & $84(32 \%)$ & $61(36 \%)$ & $23(25 \%)$ & 0.08 \\
\hline Type 2 diabetes (\%) & $67(26 \%)$ & $50(30 \%)$ & $17(19 \%)$ & 0.06 \\
\hline Stroke/SE/TIA (\%) & $41(16 \%)$ & $31(18 \%)$ & $10(11 \%)$ & 0.12 \\
\hline Vascular disease (\%) & $15(6 \%)$ & $13(8 \%)$ & $2(2 \%)$ & 0.07 \\
\hline $\begin{array}{l}\text { Excessive alcohol } \\
\text { intake }(\%)^{\wedge}\end{array}$ & $43(17 \%)$ & $29(18 \%)$ & $14(16 \%)$ & 0.73 \\
\hline $\begin{array}{l}\mathrm{CHA}_{2} \mathrm{DS}_{2} \text {-VASc score } \\
\geq 3(\%) \dagger\end{array}$ & $185(71 \%)$ & $130(77 \%)$ & $55(60 \%)$ & $<0.01$ \\
\hline \multicolumn{5}{|l|}{ Prescribed therapies } \\
\hline Anticoagulant (\%) & $141(54 \%)$ & $91(54 \%)$ & $50(55 \%)$ & 0.87 \\
\hline $\begin{array}{l}\text { Clopidogrel+aspirin } \\
(\%)\end{array}$ & $46(18 \%)$ & $33(20 \%)$ & $13(14 \%)$ & 0.29 \\
\hline Aspirin only (\%) & $126(49 \%)$ & $87(52 \%)$ & $39(43 \%)$ & 0.19 \\
\hline Digoxin (\%) & $92(35 \%)$ & $69(41 \%)$ & $23(25 \%)$ & 0.01 \\
\hline$\beta$-Blocker (\%) & $130(50 \%)$ & $87(52 \%)$ & $43(47 \%)$ & 0.52 \\
\hline Anti-arrhythmic (\%) & $69(27 \%)$ & $41(24 \%)$ & $28(31 \%)$ & 0.26 \\
\hline
\end{tabular}

For the current research, the $\mathrm{CHA}_{2} \mathrm{DS}_{2}$-VASc score is scored from 0 to 8 (not 0 to 9 as is the norm) due to the exclusion of patients with chronic heart failure.

*Assessed in $\mathrm{n}=257$; $\wedge$ More than two standard drinks on any occasion. Assessed in $\mathrm{n}=250$ patients.

$+C H A_{2} D_{2}$-VASC score definition: $C_{1}$ congestive heart failure/left ventricular dysfunction (one point); $\mathrm{H}$, hypertension (even if treated; one point); $\mathrm{A}_{2}$, age $\geq 75$ years (two points); $D$, diabetes mellitus (one point); $S_{2}$, stroke/SE/TIA (two points); $V$, vascular disease (one point); $A$, age $65-74$ years (one point); Sc, sex category (female sex; one point).

$A F$, atrial fibrillation; CAD, coronary artery disease; $M C l$, mild cognitive impairment; MoCA, Montreal Cognitive Assessment; SAFETY, Standard versus Atrial Fibrillation

spEcific managmenT studY; SE, systemic embolism; TIA, transient ischaemic attack. care or a home-based, multidisciplinary, AF-specific intervention designed to reduce morbidity and mortality.

Wherever feasible, we prospectively measured cognitive function using the Montreal Cognitive Assessment (MoCA) tool that was designed to be more sensitive and specific to MCI. ${ }^{16}$ The MoCA was applied at the point of recruitment in hospital to all eligible patients. Ethics approval was obtained from the Central Northern Adelaide Health Service Ethics of Human Research Committee, Metro South Health Service District Human Research Ethics Committee, Melbourne Health Human Research Ethics Committee, Western Health Office for Research and the ACT Health Directorate Human Research Ethics Committee. Written informed consent was obtained from each study participant before study procedures were conducted.

\section{Participants}

A systematic screening programme to identify eligible inpatients was conducted at each participating hospital. Patients were approached for recruitment if they had a documented diagnosis of recurrent paroxysmal (ie, recurrent episodes by history as documented on ECG), persistent or permanent AF; were living independently in the community or their own home posthospitalisation; and were able and willing to provide written informed consent to participate. Patients were excluded if they were aged less than 45 years, had a primary diagnosis of valvular heart disease, were scheduled for catheter ablation, had preexisting chronic heart failure (CHF) as evidenced by the combination of symptoms indicative of New York Heart Association class III-IV with a documented left ventricular ejection fraction less than $45 \%$, or had a transient form of AF. Patients determined to be demented, as assessed on routine evaluations conducted by hospital clinical teams, were also excluded.

\section{Data collection}

Baseline profiling

A comprehensive range of sociodemographic and clinical data were collected at baseline. This included basic demographic characteristics (age and sex), education (level of education completed and number of years), subtype of AF, comorbid conditions and $\mathrm{CHA}_{2} \mathrm{DS}_{2}$-VASc score to identify those at very high thromboembolic risk (with a score $\geq 3$ ). ${ }^{17}$ The $\mathrm{CHA}_{2} \mathrm{DS}_{2}$-VASc score is a validated risk stratification tool used to assess the thomboembolic risk of individuals with $\mathrm{AF}$ and indicates antithombotic therapy requirements. ${ }^{17}$ The scoring of eight criteria contributes to the final score of 0-9. Each criterion accounts for one or two points: congestive heart failure or left ventricular dysfunction (one point); hypertension (one point); age 75 years or greater (two points); diabetes mellitus (one point); stroke/ TIA/systemic embolism (two points); vascular disease (one point); age 65-74 years (one point); sex category (one point if a woman). ${ }^{17}$

\section{Assessment of cognitive function}

The MoCA is a validated screening tool for cognitive dysfunction that is administered during in-person interview in the clinical setting. The MoCA is designed to delineate MCI patients from those with normal cognition with a greater predictive value than the MMSE. ${ }^{16}$ Scoring ranges from 0 to 30 and a score of less than 26 is indicative of MCI. Six domains of cognitive function are assessed using the MoCA: executive functioning, visuospatial abilities, language abilities, short-term memory, sustained attention and orientation. Adjustment is made for obtaining a formal education of 12 years or less. Personnel conducting the MoCA were trained according to standardised 
criteria relating to questionnaire administration, question delivery and scoring. ${ }^{16}$ Given recent suggestions that a MoCA score less than 24 increases specificity for MCI without impacting on sensitivity (albeit in a younger cohort of individuals with a much lower prevalence of MCI than expected in the SAFETY cohort), ${ }^{18}$ we compared the two cut-off scores $(<26$ vs $<24)$ to generate a more conservative prevalence estimate in this cohort.

From a total of 335 SAFETY participants, 260 (78\%) were administered the MoCA at baseline (during inpatient stay) to assess cognitive function. For the purpose of this substudy, individuals whose primary language was not English $(n=17,5 \%)$ were excluded on the basis of language proficiency affecting MoCA responses, ${ }^{19}$ and those who were too unwell at the time when testing was attempted were also excluded $(n=58,17 \%)$.

\section{Statistical analyses}

Descriptive values are presented as mean $( \pm S D)$ for continuous variables or a proportion for categorical variables. Unadjusted odds ratios (ORs) with 95\% confidence intervals (CIs) compared education, AF subtype and comorbidities between patients with and without MCI. Multivariate analyses to determine independent correlates of MCI (referred to here as predictors) were examined with a stepwise linear regression model and adjusted for age, sex, AF subtype, high-risk alcohol use, comorbid conditions (hypertension, type 2 diabetes, cerebrovascular disease, coronary artery disease; CAD) and 'new AF' diagnosis (ie, first diagnosis of a chronic form of AF (recurrent paroxysmal, persistent or permanent) not related to a transient physiological state). Further categorisation and analysis of $\mathrm{CHA}_{2} \mathrm{DS}_{2}$-VASc scores was undertaken to understand which score(s) confers the independent association with MCI. Data were analysed using SPSS, V.20. A probability value of $\mathrm{p}<0.05$ (two-sided) was considered statistically significant.

\section{RESULTS}

\section{Baseline profile}

The baseline patient profile $(n=260)$ is presented in table 1 according to the presence/absence of MCI (cut-off MoCA score <26). Overall, $137(53 \%)$ participants were men with a mean age of $72 \pm 11$ years (more patients with MCI were aged $\geq 75$ years; $\mathrm{p}<0.01)$ and $80(31 \%)$ had 12 years or less of formal education. The majority of participants assessed for MCI had persistent AF $(n=224 ; 86 \%)$ with 185 (71\%) recording a $\mathrm{CHA}_{2} \mathrm{DS}_{2}$-VASc score of 3 or greater, indicating a high inherent risk of stroke. The comorbid profile of these patients was extensive: 188 (72\%), 84 (32\%) and 67 (26\%) had hypertension, CAD and type 2 diabetes, respectively. A previous history of stroke/systemic embolism/TIA was present in 41 (16\%) patients. AF-specific therapies were prescribed in similar proportions. However, digoxin was significantly more likely to be prescribed to patients displaying MCI $(\mathrm{p}=0.01)$.

\section{Baseline cognitive function}

The overall mean MoCA score at baseline (prehospital discharge) in this cohort was $23 \pm 4$; equivalent scores being $21 \pm 3$ versus $27 \pm 1$ in those with and without identified MCI, respectively. Overall, 169 patients $(65 \%$, 95\% CI 59\% to $71 \%)$, of which 53\% were men, recorded a MoCA score less than 26. As shown in table 1 , on an unadjusted basis, those with MCI were on average 6 years older than those without MCI $(74 \pm 10$ years vs $68 \pm 12$ years, $p<0.001)$. A higher proportion of individuals with MCI had less than 12 years of formal education (despite correction for education level). Those classified with MCI also had an increased likelihood of concurrent hypertension (OR
1.61, 95\% CI 0.92 to 2.82 ), CAD (OR 1.67, 95\% CI 0.95 to 2.95 ), type 2 diabetes (OR 1.83, 95\% CI 0.98 to 3.41) and stroke/systemic embolism/TIA (OR $1.82,95 \%$ CI 0.85 to 3.91 ). Consequently, those classified as having MCI also had a higher risk of thromboembolic events with a 2-fold increased likelihood of a higher $\mathrm{CHA}_{2} \mathrm{DS}_{2}$-VASc score of 3 or greater (OR $2.18,95 \%$ CI 1.26 to 3.79$)$.

\section{MoCA sensitivity analyses}

When applying a lower (more stringent) MoCA cut-off score of less than 24, the proportion of those classified as MCI fell from $65 \%(95 \%$ CI $59 \%$ to $71 \%$; cut-off $<26)$ to $49 \%(95 \%$ CI $43 \%$ to $55 \%$ ). Patients classified with MCI on this basis had a mean age of $75 \pm 10$ years (only slightly older than the original MCI group) and a mean MoCA score of $20 \pm 3$ compared to 69 \pm 12 years and $27 \pm 2$ for those without MCI, respectively. In both MCI groups, $53 \%$ were men.

\section{Affected cognitive domains}

Tables 2 and 3 outline the proportion of patients in each group (with or without $\mathrm{MCI}$ ) demonstrating paired deficits in each affected cognitive domain at the time of testing, as measured by the MoCA. In both groups, high levels of dysfunction were identified in almost all cognitive domains (most notably in executive functioning, visuospatial and short-term memory domains); however, dysfunction in orientation was only seen in $25 \%$ and $2 \%$ of individuals with and without MCI, respectively. Overall, deficits were more extensive in those with MCI. In total, only 11 individuals obtained a 'perfect' MoCA score of 30 .

\section{Predictors of $\mathrm{MCl}$}

Table 4 shows the independent predictors of MCI. MCI was more likely in those reporting lower levels of education (including $<8$ years education or trade qualifications) and in those with a higher $\mathrm{CHA}_{2} \mathrm{DS}_{2}$-VASc score. The odds of MCI increased by $46 \%$ for every unit increase in the $\mathrm{CHA}_{2} \mathrm{DS}_{2}$-VASc score. In addition, those prescribed digoxin were more likely to demonstrate MCI. Further analysis of a subgroup of patients $(n=32)$ who had digoxin levels tested at the time of MoCA administration revealed a (non-significant) trend towards those with $\mathrm{MCI}$ having, on average, a lower body mass index $\left(28.5 \pm 6.6 \mathrm{~kg} / \mathrm{m}^{2}\right)$ but higher serum digoxin levels $(0.96 \pm 0.34 \mu \mathrm{g} / \mathrm{l})$ in contrast to those without MCI who had, on average, a higher body mass index $\left(30.0 \pm 5.7 \mathrm{~kg} / \mathrm{m}^{2}\right)$ and lower serum digoxin levels $(0.45$ $\pm 0.08 \mu \mathrm{g} / \mathrm{l})$. Figure 1 shows a more detailed description of the relationship between the $\mathrm{CHA}_{2} \mathrm{DS}_{2}$-VASc score and MCI. An incremental decrease in the MoCA score (ie, an increased likelihood of demonstrating $\mathrm{MCI}$ ) with higher $\mathrm{CHA}_{2} \mathrm{DS}_{2}$-VASc scores was shown.

\section{DISCUSSION}

Consistent with the high potential for cognitive decline and vascular dementia ${ }^{7}$ in a typical cohort of 260 older individuals hospitalised with chronic AF, we found that a high proportion of our study cohort (approximately two-thirds) displayed concurrent MCI. Even with a more stringent definition (ie, lowering the qualifying MoCA score to $<24$ ) the underlying prevalence of MCI was approximately half. Deficits in executive functioning, visuospatial abilities and short-term memory were common, as were overlapping deficits across multiple cognitive domains. Those found to have MCI were more likely to have lower education levels and the highest thromboembolic risk (primarily calculated on the basis of more advanced age and cardiovascular 
Table 2 Proportion of patients with $\mathrm{MCl}(\mathrm{n}=169)$ demonstrating deficits within each cognitive domain

\begin{tabular}{|c|c|c|c|c|c|c|}
\hline & $\begin{array}{l}\text { Executive functioning } \\
\mathrm{n}=155(92 \%)\end{array}$ & $\begin{array}{l}\text { Visuospatial abilities } \\
n=142(84 \%)\end{array}$ & $\begin{array}{l}\text { Language abilities } \\
\mathrm{n}=107(63 \%)\end{array}$ & $\begin{array}{l}\text { Short-term memory } \\
n=169(100 \%)\end{array}$ & $\begin{array}{l}\text { Sustained attention } \\
n=116(69 \%)\end{array}$ & $\begin{array}{l}\text { Orientation } \\
n=42(25 \%)\end{array}$ \\
\hline Executive functioning & $155 / 155(100 \%)$ & & & & & \\
\hline Visuospatial abilities & $132 / 155(85 \%)$ & $142 / 142(100 \%)$ & & & & \\
\hline Language abilities & $98 / 155(63 \%)$ & $94 / 142(66 \%)$ & $107 / 107(100 \%)$ & & & \\
\hline Short-term memory & $155 / 155(100 \%)$ & $142 / 142(100 \%)$ & 107/107 (100\%) & $169 / 169$ (100\%) & & \\
\hline Sustained attention & $107 / 155(69 \%)$ & $103 / 142(73 \%)$ & $75 / 107(70 \%)$ & $116 / 169(69 \%)$ & $116 / 116(100 \%)$ & \\
\hline Orientation & $40 / 155(26 \%)$ & $32 / 142(23 \%)$ & $30 / 107(28 \%)$ & $42 / 169(25 \%)$ & $30 / 116(26 \%)$ & $42 / 42(100 \%)$ \\
\hline
\end{tabular}

insults) while being most likely to be prescribed agents with the narrowest benefit-to-risk ratios.

As such, these data confirm previous studies suggesting high levels of cognitive dysfunction in those with $\mathrm{AF}^{8}{ }^{8}$ They also represent an important clinical warning, particularly given that the risk factors we identified are frequently seen in those being treated for AF. The role of digoxin, a commonly prescribed drug in the presence of $\mathrm{AF}$, but with clear potential for toxicity and associated mental confusion in older individuals, ${ }^{20} 21$ in potentially contributing to $\mathrm{MCI}$ in this cohort is also an important finding. Low digoxin levels $(0.5-0.8 \mu \mathrm{g} / \mathrm{l})$ have been shown to be associated with better clinical outcomes (including cognitive functioning) and lower mortality. ${ }^{21}$ However, cognitive impairment has also been found to occur at levels of serum digoxin that are within the therapeutic range. ${ }^{20} 22$ In the current study, those with MCI and concurrent digoxin levels had lower body weight but greater digoxin levels, potentially reflecting higher tissue saturation and, thus, digoxin toxicity albeit at a therapeutic level. Recommendations have been made outlining the need for digoxin withdrawal in any patient displaying cognitive symptoms, even when the serum digoxin level is within the normal therapeutic range. ${ }^{20}$

Our findings are of obvious clinical concern in AF due to the heavy reliance on self-care behaviours crucial to managing this complex chronic disease successfully. Inadequate self-care has been identified as a major contributor to hospital readmission and to the poor outcomes associated with heart failure; ${ }^{23}$ the same can be assumed for AF. Cause and effect is difficult to delineate, however. Individuals with MCI may have problems performing complex tasks such as preparing meals or paying bills while still being able to perform activities of daily living (and thus maintain a sense of independence). ${ }^{24}$ The ability to learn and acquire knowledge is also influenced by MCI, and lack of knowledge about chronic disease and self-care has been shown to result in poor self-care maintenance and management behaviours. $^{25}$ Intact executive functions have been identified as crucial for the management of chronic conditions. ${ }^{26}$ Impaired executive functioning limits an individual's ability to recognise symptoms and make decisions related to symptom management. ${ }^{27}$ These were all factors that influenced the subsequent management of those allocated to the intervention arm of SAFETY - with incremental surveillance and support applied according to a traffic-light classification system ${ }^{28}$ that 'titrates' post-discharge support in this context using more than just clinical parameters to determine an increased risk of suboptimal health outcomes. In this case, the presence of MCI signified a need to modify expectations of 'self-care' and increase the role of caregivers and other healthcare providers (eg, incremental community pharmacist support and/or use of medicine reminder tools). Whether this was sufficiently robust in terms of additional support will be a major factor in determining the overall success of the home-based, nurse-led intervention being compared to usual post-discharge care. ${ }^{15}$

Beyond the impact of the SAFETY intervention, these data have immediate clinical implications. Screening of MCI can and should be used as a risk delineation strategy to add to the suite of risk stratification tools available for the assessment of patients with AF. Pitfalls of therapy, clinical lability or adverse behaviours can be identified and highlighted, triggering increased patient surveillance for those at high risk of instability or events. Increased surveillance is often required to promote treatment adherence and self-care management. In addition, education focussed on addressing peripheral risk factors can be tailored according to individual needs. Furthermore, AF-specific interventions should be focused on strengthening the areas of cognition most commonly affected. In their recent systematic review,

Table 3 Proportion of patients without $\mathrm{MCl}(n=91)$ demonstrating deficits within each cognitive domain

\begin{tabular}{|c|c|c|c|c|c|c|}
\hline & $\begin{array}{l}\text { Executive functioning } \\
\mathrm{n}=45(49 \%)\end{array}$ & $\begin{array}{l}\text { Visuospatial abilities } \\
\mathrm{n}=36(40 \%)\end{array}$ & $\begin{array}{l}\text { Language abilities } \\
\mathrm{n}=27(30 \%)\end{array}$ & $\begin{array}{l}\text { Short-term memory } \\
\mathrm{n}=68(75 \%)\end{array}$ & $\begin{array}{l}\text { Sustained attention } \\
n=16(18 \%)\end{array}$ & $\begin{array}{l}\text { Orientation } \\
n=2(2 \%)\end{array}$ \\
\hline Executive functioning & $45 / 45(100 \%)$ & & & & & \\
\hline Visuospatial abilities & $21 / 45(47 \%)$ & $36 / 36(100 \%)$ & & & & \\
\hline Language abilities & $16 / 45(36 \%)$ & $6 / 36(17 \%)$ & $27 / 27(100 \%)$ & & & \\
\hline Short-term memory & $35 / 45(78 \%)$ & $24 / 36(67 \%)$ & $21 / 27(78 \%)$ & $68 / 68(100 \%)$ & & \\
\hline Sustained attention & $10 / 45(22 \%)$ & $6 / 36(17 \%)$ & $7 / 27(26 \%)$ & $11 / 68(16 \%)$ & $16 / 16(100 \%)$ & \\
\hline Orientation & $1 / 45(2 \%)$ & $0 / 36(0 \%)$ & $0 / 27(0 \%)$ & $2 / 68(3 \%)$ & $0 / 16(0 \%)$ & $2 / 2(100 \%)$ \\
\hline
\end{tabular}


Table 4 Independent predictors of $\mathrm{MCl}$ (age and sex adjusted)

\begin{tabular}{lcll}
\hline Variable & $\mathbf{p}$ Value & OR & $95 \% \mathrm{Cl}$ \\
\hline Education level overall & $<0.01$ & - & - \\
$\quad$ Tertiary education (reference) & - & 1.00 & - \\
Secondary school & 0.11 & 1.79 & 0.88 to 3.64 \\
Technical/trade school & $<0.01$ & 6.00 & 2.07 to 17.42 \\
Primary school & $<0.01$ & 5.29 & 1.95 to 14.36 \\
$\mathrm{CHA}_{2} \mathrm{DS}_{2}$-VASc score (per unit increment) & $<0.001$ & 1.46 & 1.23 to 1.74 \\
Digoxin treatment vs rest & 0.01 & 2.19 & 1.17 to 4.10 \\
Age & 0.69 & 1.01 & 0.97 to 1.04 \\
Male sex & 0.20 & 1.49 & 0.81 to 2.73 \\
\hline $\mathrm{MCl}$, mild cognitive impairment. & & &
\end{tabular}

Eggermont $e t a l^{6}$ suggested that MCI may be addressed simply by optimising cardiac treatment. The incorporation of physical activity programmes into interventions has also been suggested due to positive improvements in cognition observed in patients with CAD and chronic heart failure. ${ }^{6}{ }^{29}$ In addition to the screening and detection of MCI, identifying associated independent predictors of lower education level, higher thromboembolic risk and the use of digoxin allows the potential to address or increase surveillance on these peripheral factors in an attempt to prevent the development of MCI and vascular dementia in those who present with AF.

There are a number of limitations that require comment. First, we applied only one clinical assessment tool to assess cognitive function. While the MoCA has been shown to be more sensitive and specific at discerning cognitive deficits than the MMSE, more extensive psychometric testing in addition to the testing of functional capacity would be required to confirm the presence of MCI. ${ }^{18}$ However, the MoCA could reasonably constitute an initial screening of MCI due to its short length (approximately $10 \mathrm{~min}$ ) and ease of administration. It is also publicly available for use. The decision to use less than 26 as the cut-off score for defining MCI also influences this research, particularly when such a large decrease in the proportion of classified MCI within this population was observed in the sensitivity analysis. Whether a more stringent cut-off of less than 24 is required for more specific determination of the prevalence in these patients should be the subject of further research. The exclusion of non-English-speaking individuals may have introduced selection bias, although English

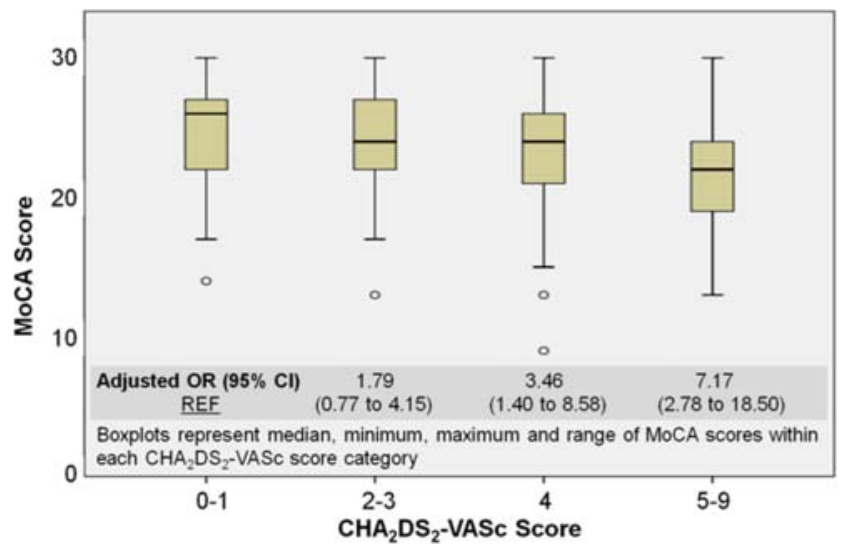

Figure 1 Correlation between $\mathrm{CHA}_{2} \mathrm{DS}_{2}$-VASC and Montreal Cognitive Assessment (MoCA) scores. This figure is only reproduced in colour in the online version. competency is necessary for comprehensive understanding of the test questions. Furthermore, the often chaotic acute clinical setting under which the MoCA was conducted in addition to the patients' altered health state may not represent an ideal testing situation. Moreover, the duration and cause of $\mathrm{MCI}$ is not known and cannot be distinguished in this cohort. Finally, despite the selection of a 'real world' cohort, we cannot discount the possibility of bias in study selection and these data may not readily apply to other hospitalised cohorts.

Despite these limitations, these data have important clinical implications for the post-discharge management of patients with chronic AF. We found that MCI is highly prevalent in typically older patients who have been hospitalised with chronic AF before discharge. Overall, cognitive deficits are abundant in this population. Beyond education levels, MCI correlated with increasing thromboembolic risk and the use of digoxin. In addition, residual levels of MCI remain high in these individuals post-discharge. Therefore, we would recommend that patients with AF should be routinely screened for MCI (as a potential source of risk delineation), and subsequent disease management programmes implemented in the setting of $\mathrm{AF}$ (as has been recommended in relation to heart failure management) ${ }^{30}$ should be modulated if $\mathrm{MCI}$ is detected.

\section{CONCLUSION}

$\mathrm{MCI}$ is common in high-risk patients hospitalised with chronic $\mathrm{AF}$ and is potentially related to poorer health outcomes. Assessment of patients with chronic AF should include $\mathrm{MCI}$ screening using the MoCA, an easily administered and effective tool that identifies patients for whom more intensive surveillance is required to maintain clinical stability and optimise management.

Contributors All authors contributed to the study design, data analysis, manuscript writing, reviewing and editing

Funding The SAFETY study is funded by a National Health and Medical Research Council of Australia Programme Grant (519823). In addition, JB, MJC and SS are supported by the National Health and Medical Research Council of Australia. The study was supported in part by the Victorian government's operational infrastructure support programme. The National Health and Medical Research Council of Australia and the Victorian Government had no involvement in the study design; data collection, analysis and interpretation; or writing or preparation of this manuscript for publication.

Competing interests None.

Ethics approval This study was approved by the human research ethics committee(s) associated with each of the study sites (hospitals).

Patient consent Obtained.

Provenance and peer review Not commissioned; internally peer reviewed.

\section{REFERENCES}

1 Go AS, Hylek EM, Phillips KA, et al. Prevalence of diagnosed atrial fibrillation in adults: national implications for rhythm management and stroke prevention: the AnTicoagulation and Risk Factors in Atrial Fibrillation (ATRIA) Study. JAMA 2001;285:2370-5.

2 Benjamin EJ, Wolf PA, D'Agostino RB, et al. Impact of atrial fibrillation on the risk of death: the Framingham Heart Study. Circulation 1998;98:946-52.

3 Wolf PA, Abbott RD, Kannel WB. Atrial fibrillation as an independent risk factor for stroke: the Framingham Study. Stroke 1991;22:983-8.

4 Wang TJ, Larson MG, Levy D, et al. Temporal relations of atrial fibrillation and congestive heart failure and their joint influence on mortality: the Framingham Heart Study. Circulation 2003;107:2920-5.

5 Rathore SS, Berger AK, Weinfurt KP, et al. Acute myocardial infarction complicated by atrial fibrillation in the elderly - prevalence and outcomes. Circulation 2000;101:969-74.

6 Eggermont LHP, de Boer K, Muller M, et al. Cardiac disease and cognitive impairment: a systematic review. Heart 2012;98:1334-40. 
7 Ott A, Breteler MM, de Bruyne MC, et al. Atrial fibrillation and dementia in a population-based study. The Rotterdam Study. Stroke 1997;28:316-21.

8 Knecht S, Oelschlager C, Duning T, et al. Atrial fibrillation in stroke-free patients is associated with memory impairment and hippocampal atrophy. Eur Heart J 2008;29:2125-32.

9 Kilander L, Andren B, Nyman $\mathrm{H}$, et al. Atrial fibrillation is an independent determinant of low cognitive function: a cross-sectional study in elderly men. Stroke 1998;29:1816-20.

10 Ezekowitz MD, James KE, Nazarian SM, et al. Silent cerebral infarction in patients with nonrheumatic atrial fibrillation. Circulation 1995;92:2178-82.

11 Feinberg WM, Seeger JF, Carmody RF, et al. Epidemiologic features of asymptomatic cerebral infarction in patients with nonvalvular atrial fibrillation. Arch Intern Med 1990;150:2340-4.

12 Tarvonen RI, Kurki T, Rajala T, et al. Relationship between vascular factors and white matter low attenuation of the brain. Acta Neurol Scand 1993;87:286-9.

13 Petersen RC. Mild cognitive impairment. N Engl J Med 2011;364:2227-34.

14 Kumar R, Dear KBG, Christensen $\mathrm{H}$, et al. Prevalence of mild cognitive impairment in 60- to 64-year-old community-dwelling individuals: the personality and total health through life 60+ study. Dement Geriatr Cogn Disord 2005; 19:67-74

15 Carrington MJ, Ball J, Horowitz JD, et al. Navigating the fine line between benefit and risk in chronic atrial fibrillation: Rationale and design of the Standard versus Atrial Fibrillation spEcific managmenT studY (SAFETY). Int I Cardiol 2011; In Press.

16 Nasreddine ZS, Phillips NA, Bedirian V, et al. The Montreal Cognitive Assessment, MoCA: a brief screening tool for mild cognitive impairment. J Am Geriatr Soc 2005;53:695-9.

17 Lip GY, Nieuwlaat R, Pisters R, et al. Refining clinical risk stratification for predicting stroke and thromboembolism in atrial fibrillation using a novel risk factor-based approach: the Euro Heart survey on atrial fibrillation. Chest 2010;137:263-72.

18 McLennan SN, Mathias JL, Brennan LC, et al. Validity of the montreal cognitive assessment (MoCA) as a screening test for mild cognitive impairment (MCI) in a cardiovascular population. J Geriatr Psychiatry Neurol 2011;24:33-8.
19 McLennan SN, Mathias JL, Brennan LC, et al. Cognitive impairment predicts functional capacity in dementia-free patients with cardiovascular disease. J Cardiovasc Nurs 2010;25:390-7.

20 Wofford JL, Ettinger WH. Risk factors and manifestations of digoxin toxicity in the elderly. Am J Emerg Med 1991:9:11-15; discussion 33-4.

21 Bauman JL, Didomenico RJ, Galanter WL. Mechanisms, manifestations, and management of digoxin toxicity in the modern era. Am J Cardiovasc Drugs 2006:6:77-86.

22 Eisendrath SJ, Sweeney MA. Toxic neuropsychiatric effects of digoxin at therapeutic serum concentrations. Am J Psychiatry 1987;144:506-7.

23 Schwarz KA, Elman CS. Identification of factors predictive of hospital readmissions for patients with heart failure. Heart Lung 2003;32:88-99.

24 Davis KK, Mintzer M, Dennison HCR, et al. Targeted intervention improves knowledge but not self-care or readmissions in heart failure patients with mild cognitive impairment. Eur J Heart Fail 2012;14:1041-9.

25 van der Wal MH, Jaarsma T, Moser DK, et al. Compliance in heart failure patients: the importance of knowledge and beliefs. Eur Heart J 2006;27:434-40.

26 Naik $A D$, Dyer $C B$, Kunik ME, et al. Patient autonomy for the management of chronic conditions: a two-component re-conceptualization. Am J Bioeth 2009;9:23-30.

27 Cameron J, Worrall-Carter L, Page K, et al. Does cognitive impairment predict poor self-care in patients with heart failure? Eur J Heart Fail 2010;12:508-15.

28 Carrington MJ, Kok S, Jansen K, et al. The Green, Amber, Red Delineation of Risk and Need (GARDIAN) management system: a pragmatic approach to optimizing heart health from primary prevention to chronic disease management. Eur J Cardiovasc Nurs. Published Online First: 29 June, 2012 doi: 10.1177/ 1474515112451702

29 Carles S Jr, Curnier D, Pathak A, et al. Effects of short-term exercise and exercise training on cognitive function among patients with cardiac disease. J Cardiopul Rehabil Prev 2007;27:395-9.

30 McLennan SN, Pearson SA, Cameron J, et al. Prognostic importance of cognitive impairment in chronic heart failure patients: does specialist management make a difference? Eur J Heart Fail 2006:8:494-501. 